\title{
Clinical characteristics of a group of deaths with COVID-19 pneumonia in Wuhan, China: retrospective case series
}

\section{Tao Yao}

Wuhan University Renmin Hospital https://orcid.org/0000-0001-8450-7201

\section{Yan Gao}

Wuhan University Renmin Hospital

Qin Cui

Wuhan University Renmin Hospital https://orcid.org/0000-0002-8165-246X

\section{Bo Peng}

Wuhan University Renmin Hospital

\section{Yan Chen}

Wuhan University Renmin Hospital

\section{Jiansheng Li}

Wuhan University Renmin Hospital

Chao Huang

Wuhan University Renmin Hospital

\section{Chunping He}

Wuhan University Renmin Hospital

Jie Pu

Wuhan University Renmin Hospital

\section{Jiajun Wei}

Wuhan University Renmin Hospital

\section{Yanqiang Zhan}

Wuhan University Renmin Hospital https://orcid.org/0000-0001-7265-7368

Jie Yan

Wuhan University Renmin Hospital

Jinghua Tian

Wuhan University Renmin Hospital

Zhaohui Zhang ( $\nabla$ zhangzhaohui2020@126.com )

Wuhan University Renmin Hospital

\section{Zhichao Liu ( $\nabla$ zhichao.liu@whu.edu.cn )}

Wuhan University Renmin Hospital https://orcid.org/0000-0001-9548-7883 


\section{Research Article}

Keywords: characteristics, COVID-19, pneumonia, death

Posted Date: March 20th, 2020

DOI: https://doi.org/10.21203/rs.3.rs-18090/v1

License: (c) (i) This work is licensed under a Creative Commons Attribution 4.0 International License. Read Full License

Version of Record: A version of this preprint was published at BMC Infectious Diseases on September 22nd, 2020. See the published version at https://doi.org/10.1186/s12879-020-05423-7. 


\section{Abstract}

Background: With the widespread outbreak of novel coronavirus diseases 2019(COVID-19), more and more death cases were reported, however, limited data are available for the patients who died. We aimed to explore the clinial characteristics of deaths with COVID-19 pneumonia

Methods: We abstracted and analyzed epidemiological, demographic, clinical, and laboratory data from 83 death cases with COVID-19 pneumonia in East hospital of Wuhan university Renmin hospital,between January 26, 2020, and February 28, 2020.

Results: Of the 83 deaths, none was the medical staff. The mean age was 71.8 years (SD 13.2; range, 3497 years) and 53(63.9\%) were male. The median from onset to admission was 10 days (IQR 7-14: range, 2-43 days), to death was 17days (IQR 14-21: range, 6-54 days). Most deaths (66[80\%]) had underlying comorbid diseases, the most of which was hypertension [47(57\%)]. The main initial symptoms of these 83 deaths were shortness of breath(98.8\%), fever(94\%) and myalgia or fatigue( $90.4 \%)$. Laboratory analyses showed the lymphocytopenia in $69(83 \%)$ deaths, hypoalbuminemia in $77(93 \%)$ deaths, the elevation of lactate dehydrogenase in $79(95 \%)$ deaths, procalcitonin in $69(83 \%)$ deaths and C-reactive protein in 79(95\%) deaths. All 83 patients received antiviral treatment, $81(97.6 \%)$ deaths received antibiotic therapy, and $54(65.1 \%)$ deaths received glucocorticoid therapy and $20(24.1 \%)$ patients received invasive mechanical ventilation.

Conclusion: Most of the deaths with COVID-19 pneumonia were elderly patients with underlying comorbid diseases, especially those over 70 years of age. The time of death was mostly 15-21 days after the onset of the disease. More care should be given to the elderly in the further prevention and control strategies of COVID-19.

\section{Background}

The novel coronavirus diseases 2019(COVID-19) first reported in Wuhan, Hubei province, China[1, 2]. It then spread widely to other regions of China and 24 other nations[3, 4]. With the widespread outbreak of COVID-19, more and more cases of death were reported[5-7]. As of February 11, 2020, China had confirmed 44672 cases of COVID-19, 1023 of them died (2.3\%), according to a report by the Chinese $\mathrm{CDC} .[8]$

At present, the early specific management of patients to reduce mortality has become an emergent issue confronting the current epidemic situation $[9,10]$. The previous study has reported the features of severe cases admitted to ICU with COVID-19 pneumonia[11]. However, limited data are available until now for the patients who died, little is known about clinical features of deaths with COVID-19 pneumonia.

In this study, we retrospectively collected and described detailed epidemiological, demographic, clinical, and laboratory characteristics of 83 deaths with COVID-19 pneumonia who had been admitted to east 
hospital Wuhan University Renmin Hospital, which was one of the first designated hospitals in Wuhan to admit severe patients with COVID-19.

\section{Methods}

\section{Study design and patients}

This is a single-center retrospective study. We reviewed all patients with COVID-19 pneumonia who were admitted to East Hospital of Wuhan University Renmin hospital as of February 28, 2020, and collected data on death cases in hospital. East Hospital of Wuhan University Renmin hospital located in Wuhan, Hubei Province, China, was designated as one of the first hospitals to admit severe adult patients with COVID-19 pneumonia by government. The diagnostic standard of COVID-19 pneumonia is based on the 4th edition protocole of the New Coronavirus Pneumonia Prevention and Control Program issued by the National Health Commission of Republic of China[12].

This study protocol complied with the Medical Ethical Committee of Wuhan University Renmin hospital (No.WDYR2020-k050). Written informed consent was waived due to the rapid emergence of this infectious disease.

\section{Data collection}

Several investigators reviewed the electronic medical record system of the hospital, and abstracted epidemiological, demographic, clinical, and laboratory data from death cases with COVID-19 pneumonia as of February 28,2020 . The other two researchers reviewed and checked the data collected. The investigators directly contacted their families to refine the data if some epidemiological date of patients were not available in the medical record.

Nasopharyngeal swabs were obtained from all patients at admission. All samples were processed at the Department of Clinical Laboratory of Wuhan university Renmin Hospital. COVID-19 was confirmed by Real-time polymerase chain reaction testing according to WHO guidelines for Laboratory testing[13]. Positive confirmed patients with COVID-19 infection were defined as at least 2 positive test results.

\section{Statistical analysis}

Continuous variables are expressed as the means \pm standard deviations (SD) if they are normally distributed or medians (interquartile ranges, IQR) if they are not. Categorical variables are expressed as frequencies and percentages. All statistical analysis was performed with SPSS, version 25.0 (SPSS Inc., Chicago, IL, USA).

\section{Results}


As of February $28,2020,83(5.7 \%)$ of the 1439 patients with COVID-19 pneumonia admitted to the hospital, died in hospital. None of the 83 deaths were medical staff, and there was no definite exposure history of patients with suspected or confirmed COVID-19.

The mean age was 71.8 years (SD 13.2; range, 34-97 years), including 26 patients over 80 years (31\%) and 2 patient under 40 years (2\%). Among the deaths, 53(63.9\%) were male. The initial symptoms of these 83 patients were shortness of breath(98.8\%), fever(94\%), myalgia or fatigue( $90.4 \%)$, anorexia( $82 \%)$, cough(60.2\%), hemoptysis(6\%), pharyngalgia(6\%), headache(3.6\%), nausea or vomiting $(2.4 \%)$ and diarrhea(4.8\%) (Table 1).

Onset-to-admission interval of the 83 deaths was between 2 and 43 days (median 10 days, IQR 7-14), most of them were 6-10 days (43\%). Onset-to-death interval was between 6 and 54 days (median 17 days, IQR 14-21), and most of them were $15-21$ days for $56 \%$ of women and $49 \%$ of men (Table 2). Figure 1 showed the date distribution of illness onset in all 83 patients. As described in this figure, the most dates of illness onset are between January 20 and January 28, 2020.

Of the 83 deaths, 66 patients (80\%) had chronic comorbidities, the most of which was hypertension(57\%), followed by cardiovascular disease(31\%), diabetes $(26 \%)$, cerebrovascular disease $(17 \%)$, chronic lung disease(19.3\%), chronic renal disease (6\%), malignancy (6\%) and chronic liver disease (4\%) (Table 3).

The main laboratory findings of the deaths on admission were shown in Table 4. The results of the blood count showed that white blood cell count in 5(6\%) patients, lymphocyte count in 69(83\%) patients, hemoglobin in $34(41 \%)$ patients, and platelets in $24(29 \%)$ patients were below the normal range. In addition, white blood cell count in $34(41 \%)$ patients, and monoclear leucocyte in $16(19 \%)$ patients were above the normal range.

On admission, many patients had an abnormal liver function and renal function. Aspartate aminotransferase in $57 \%$ patients, gamma-glutamyl transpeptidase in $49 \%$ patients, serum creatinine in $47 \%$ patients and blood urea nitrogen in $53 \%$ patients were above the normal range. Albumin was lower than the normal level in most patients $93 \%$. Lactate dehydrogenase increased in $95 \%$ patients. Most patients have abnormal coagulation function, which showed the elevation of D-dimer in $94 \%$ patients, the extension of Prothrombin time in $43 \%$ patients and Activated partial thromboplastin time in $33 \%$ patients. Moreover, procalcitonin (83\%) and C-reactive protein 95\%) increased above the normal range in most patients.

Each patient performed a chest CT scan on admission, and pneumonia was confirmed in all 83 patients, and 71 patients were involved in the bilateral lung (Table 5). Multiple patchy ground-glass shadows were the main feature in the chest CT of most patients. The typical manifestation is the patchy ground-glass shadow in the lung CT.

In drug treatment, all 83 patients received antiviral therapy for 5-10 days, and all of them received abidole, 58 received oseltamivir, 16 received ribavirin, 4 received lopinavir and ritonavir. Of 83 patients, 
81(97.6\%) patients received antibiotics therapy, 54(65.1\%) patients received glucocorticoid therapy and 39 patients received intravenous immunoglobulin therapy. In non-drug treatment, $6(7.3 \%)$ patients received continuous replacement therapy due to severe renal dysfunction, 20(24.1\%) patients received invasive mechanical ventilation, $1(1.2 \%)$ patients were given extracorporeal membrane oxygenation treatment(ECOM). (Table 5)..

\section{Discussion}

This retrospective study described the epidemiological and clinical characteristics of 83 deaths with COVID-19 pneumonia. To my knowledge, our study is the first epidemiological investigation, whose subjects were all patients with COVID-19 pneumonia who died.

In this study, the median of the onset-to-admission interval was longer than that of patients in the previous two studies $[11,14]$. Most patients were hospitalized more than 6 days after the onset of the disease, and the longest was 43 days. Two factors likely contributed to the interval. Fisrt】some patients have no severe symptoms in the early stage and it took more for home isolation and community treatment. Second, due to the COVID-19 outbreak, the isolation ward of the hospital may have been under capacity in the initial. Most of the patients who died in the 15-21days after the onset of the disease, both male and female. This result indicate that the third week may be a period of high risk of death for critically ill patients with COVID-19.

Most of the deaths with COVID-19 pneumonia were elderly patients especially those over 70 years of age, and male patients. These are consistent with a recent study[10]. The cases of COVID-19 in pregnant women have been mentioned in previous studiy[15]. Of 29 pregnant women with COVID-19 in the hospital, there are no deaths so far.

The proportion of patients with comorbidities was higher than previous studies in patients with COVID$19[10,11,14,16]$. The most common comorbidities were hypertension in our study, which was diabetes in two previous cohort studies of Middle Eastern respiratory syndrome coronavirus (MRSE-Cov) infection and severe acute respiratory syndrome coronavirus(SARS-CoV) infection[17, 18]. We observed that the majority of patients who died were also geriatric patients and those suffering from chronic comorbidities. However, some healthy people(22\%) died without complications, which were an indication of the high pathogenicity of COVID-19. The initial clinical symptoms of patients infected with COVID-19 were nonspecific. There were no significant differences in the types of initial symptoms between the deaths in our study and the recently published studies[[11, 14, 16, 19]. However, the first three symptoms in our study were shortness of breath, fever, Myalgia or fatigue. A small number of patients initially presented with gastrointestinal symptoms, such as anorexia, nausea, vomiting, and diarrhoea, which were mentioned in previous studies[14, 16].

Most of the patients in our study developed acute respiratory failure including ARDS, fatal infection, abnormal coagulation and eventually multiple organ failure, except for six who died of acute myocardial infarction. As a newly identified disease, litter is known about the pathogenic mechanism of COVID-19. 
Most of the patients who died had abnormal coagulation. Increased inflammatory markers such as procalcitonin and C-reactive, lymphopenia were a common characteristic in the patients. This series of changes is a manifestation of the immune response and maybe be a factor in poor prognosis $[11,14,18]$. In a recent fatal case report, typical features of inflammation were observed in the pulmonary pathology of the patient, whose pathological section showed interstitial mononuclear inflammatory infiltrates, dominated by lymphocytes[20]. These pathological characteristics greatly resemble those of MRSE-Cov infection and SARS-CoV infection[20-22].

Until now, no drugs have been found to be specifically effective against coronaviruses. Of the 83 patients in our study, each patient received abidol, and some patients were treated with oseltamivir, ganciclovir, lopinavir and ritonavir, but none of them had a definite therapeutic effect. In addition, radcivir is an unlisted nucleotide drug whose broad-spectrum antiviral activity has been confirmed in animal models[23, 24]. It may be a potential effective drug for patients with COVID-19.[25] Two randomized controlled clinical trials (NCT04252664; NCT04257656) to assess the safety and efficacy of radcivir are is currently underway in patients hospitalized with COVID-19 pneumonia.

The patients in this study were generally had associated with a secondary bacterial infection, followed by sepsis and septic shock. $97.6 \%$ of the patients were treated with antibiotics based on abnormal inflammatory markers and bacterial culture results. In patients with SARS and MERS, the effect of glucocorticoid therapy on prognosis is Controversial[26, 27]. However, severe patients with COVID-19 may be beneficial from glucocorticoid therapy to prevent ARDS development, based on recent studies[16, 20]. $65.1 \%$ of the patients in this study received glucocorticoid therapy. In the study, 63 patients did not receive invasive mechanical ventilation, 41 of them declined invasive mechanical ventilation, and the other 22 for unknown reasons.

Our study has several limitations. First of all, the study had a limited number of cases, with only 83 deaths. However, to our knowledge, very few case series of deaths have been reported, the data is a valuable demonstration of characteristics of deaths with COVID-19 pneumonia in the early period of exponential growth. Secondly, some data such as cytokines (eg, IL2, IL4, IL6, IL10, TNF, IFN Y) were absent in patients admitted early, which were related to lung injury in previous studies SARS-CoV and MERS-CoV[28, 29]. We will routinely observe the changes of cytokines of patients in further study. Thirdly, data on all patients with COVID-19 pneumonia in the hospital during the same period were not fully available at the time of our analysis. However, this is a series of study designs and the patients will continue to be followed up.

\section{Conclusions}

This single-center retrospective case series early shows the epidemiological and clinical characteristics of deaths with COVID-19 pneumonia. Most of the deaths with COVID-19 pneumonia were elderly patients with underlying comorbid diseases, especially those over 70 years of age. The time of death was mostly 
15-21 days after the onset of the disease. More care should be given to the elderly in the further prevention and control strategies of COVID-19.

\section{Abbreviations}

novel coronavirus diseases: 2019(COVID-19); SD: standard deviations; IQR: interquartile ranges; CRRT: continuous renal replacement therapy; ECMO: extracorporeal membrane oxygenation; MRSE-Cov: Middle Eastern respiratory syndrome coronavirus; SARS-CoV: severe acute respiratory syndrome coronavirus; WHO: World health organization

\section{Declarations}

\section{Acknowledgements}

We thank all patients and their families involved in the study.

\section{Authors' contributions}

TY, YG and QC contributed equally to this article. ZCL and ZHZ conceived and designed the study. YG, QC, YC, JSL, CPH, CH, JP, JJW, YQZ, JY, and JHT collected the data. YG, and QC performed the statistical analysis. BP took responsibility for obtaining ethical approval. TY were the main contributors in writing the first draft. YG and QC revised the final manuscript. All authors read and approved the final manuscript.

\section{Funding}

No funding.

\section{Availability of data and materials}

The data will be available from the corresponding author on a reasonable request. After the publication of this study, the participant data without names an identifiers will be made available after approval from the corresponding authors and Wuhan University Renmin Hospital.

\section{Ethics approval and consent to participate}

This study protocol complied with the Medical Ethical Committee of Wuhan University Renmin hospital (No.WDYR2020-k050). Written informed consent was waived due to the rapid emergence of this infectious disease with the permission of the Medical Ethical Committee. 


\section{Consent for publication}

Not applicable.

\section{Competing interests}

We declare that we have no conflicts of interest.

\section{Author details}

${ }^{1}$ Union Department of Infection Disease, Wuhan University Renmin Hospital, Wuhan, 430060, China

${ }^{2}$ Department of Neurology, Wuhan University Renmin Hospital, Wuhan, 430060, China

${ }^{3}$ Department of Gastroenterology, Wuhan University Renmin Hospital, Wuhan, 430060, China

${ }^{4}$ Department of Critical Care Medicine, Wuhan University Renmin Hospital, Wuhan, 430060, China

${ }^{5}$ Department of Obstetrics and Gynecology, Wuhan University Renmin Hospital, Wuhan, 430060, China

\section{References}

1. Avramovski P, Avramovska M, Sikole A: Bone Strength and Arterial Stiffness Impact on Cardiovascular Mortality in a General Population. Journal of osteoporosis 2016, 2016:7030272.

2. Lu H, Stratton CW, Tang Y-W: Outbreak of pneumonia of unknown etiology in Wuhan, China: The mystery and the miracle. J Med Virol 2020, 92(4):401-402.

3. Burki T: Outbreak of coronavirus disease 2019. The Lancet Infectious Diseases 2020, 20(3):292-293.

4. Wang C, Horby PW, Hayden FG, Gao GF: A novel coronavirus outbreak of global health concern. Lancet (London, England) 2020, 395(10223):470-473.

5. Jernigan DB: Update: Public Health Response to the Coronavirus Disease 2019 Outbreak - United States, February 24, 2020. MMWR Morbidity and mortality weekly report 2020, 69(8):216-219.

6. Day M: Covid-19: Italy confirms 11 deaths as cases spread from north. BMJ (Clinical research ed) 2020, 368:m757.

7. SQ D, HJ P: Characteristics of and Public Health Responses to the Coronavirus Disease 2019 Outbreak in China. Journal of clinical medicine 2020, 9(2).

8. Wu Z, McGoogan JM: Characteristics of and Important Lessons From the Coronavirus Disease 2019 (COVID-19) Outbreak in China: Summary of a Report of 72314 Cases From the Chinese Center for Disease Control and Prevention. Jama 2020. 
9. Wei M, Yuan J, Liu Y, Fu T, Yu X, Zhang ZJ: Novel Coronavirus Infection in Hospitalized Infants Under 1 Year of Age in China. Jama 2020.

10. Yang $X, Y u$ Y, Xu J, Shu H, Xia Ja, Liu H, Wu Y, Zhang L, Yu Z, Fang M et al: Clinical course and outcomes of critically ill patients with SARS-CoV-2 pneumonia in Wuhan, China: a single-centered, retrospective, observational study. The Lancet Respiratory Medicine 2020.

11. Huang C, Wang Y, Li X, Ren L, Zhao J, Hu Y, Zhang L, Fan G, Xu J, Gu X et al: Clinical features of patients infected with 2019 novel coronavirus in Wuhan, China. Lancet (London, England) 2020, 395(10223):497-506.

12. National Health Commission of China. New coronavirus pneumonia prevention and control program (4th edn). 2020. http://www.gov.cn/zhengce/zhengceku/2020-

01/28/5472673/files/0f96c10cc09d4d36a6f9a9f0b42d972b.pdf Accessed March 15, 2020.

13. WHO. Laboratory testing for 2019 novel coronavirus (2019-nCoV) in suspected human cases. 2020. https://www.who.int/publications-detail/laboratory-testing-for-2019-novel-coronavirus-in-suspectedhuman-cases-20200117 Accessed March 15, 2020.

14. Wang D, Hu B, Hu C, Zhu F, Liu X, Zhang J, Wang B, Xiang H, Cheng Z, Xiong Y et al: Clinical Characteristics of 138 Hospitalized Patients With 2019 Novel Coronavirus-Infected Pneumonia in Wuhan, China. Jama 2020.

15. Chen H, Guo J, Wang C, Luo F, Yu X, Zhang W, Li J, Zhao D, Xu D, Gong Q et al: Clinical characteristics and intrauterine vertical transmission potential of COVID-19 infection in nine pregnant women: a retrospective review of medical records. The Lancet 2020, 395(10226):809-815.

16. Chen N, Zhou M, Dong X, Qu J, Gong F, Han Y, Qiu Y, Wang J, Liu Y, Wei Y et al: Epidemiological and clinical characteristics of 99 cases of 2019 novel coronavirus pneumonia in Wuhan, China: a descriptive study. Lancet (London, England) 2020, 395(10223):507-513.

17. Lew TWK, Kwek T-K, Tai D, Earnest A, Loo S, Singh K, Kwan KM, Chan Y, Yim CF, Bek SL et al: Acute respiratory distress syndrome in critically ill patients with severe acute respiratory syndrome. Jama 2003, 290(3):374-380.

18. Arabi YM, Arifi AA, Balkhy HH, Najm H, Aldawood AS, Ghabashi A, Hawa H, Alothman A, Khaldi A, Al Raiy B: Clinical Course and Outcomes of Critically III Patients With Middle East Respiratory Syndrome Coronavirus Infection. Annals of Internal Medicine 2014, 160(6):389-397.

19. Xu X-W, Wu X-X, Jiang X-G, Xu K-J, Ying L-J, Ma C-L, Li S-B, Wang H-Y, Zhang S, Gao H-N et al: Clinical findings in a group of patients infected with the 2019 novel coronavirus (SARS-Cov-2) outside of Wuhan, China: retrospective case series. BMJ (Clinical research ed) 2020, 368:m606.

20. Xu Z, Shi L, Wang Y, Zhang J, Huang L, Zhang C, Liu S, Zhao P, Liu H, Zhu L et al: Pathological findings of COVID-19 associated with acute respiratory distress syndrome. Lancet Respir Med 2020:S2213-2600(2220)30076-X.

21. Ng DL, Al Hosani F, Keating MK, Gerber SI, Jones TL, Metcalfe MG, Tong S, Tao Y, Alami NN, Haynes LM et al: Clinicopathologic, Immunohistochemical, and Ultrastructural Findings of a Fatal Case of 
Middle East Respiratory Syndrome Coronavirus Infection in the United Arab Emirates, April 2014. Am J Pathol 2016, 186(3):652-658.

22. Ding Y, Wang H, Shen H, Li Z, Geng J, Han H, Cai J, Li X, Kang W, Weng D et al: The clinical pathology of severe acute respiratory syndrome (SARS): a report from China. J Pathol 2003, 200(3):282-289.

23. de Wit E, Feldmann F, Cronin J, Jordan R, Okumura A, Thomas T, Scott D, Cihlar T, Feldmann H:

Prophylactic and therapeutic remdesivir (GS-5734) treatment in the rhesus macaque model of MERSCoV infection. Proc Natl Acad Sci U S A 2020:201922083.

24. Sheahan TP, Sims AC, Leist SR, Schäfer A, Won J, Brown AJ, Montgomery SA, Hogg A, Babusis D, Clarke $\mathrm{MO}$ et al: Comparative therapeutic efficacy of remdesivir and combination lopinavir, ritonavir, and interferon beta against MERS-CoV. Nat Commun 2020, 11(1):222-222.

25. Wang M, Cao R, Zhang L, Yang X, Liu J, Xu M, Shi Z, Hu Z, Zhong W, Xiao G: Remdesivir and chloroquine effectively inhibit the recently emerged novel coronavirus (2019-nCoV) in vitro. Cell Research 2020.

26. Arabi YM, Mandourah Y, Al-Hameed F, Sindi AA, Almekhlafi GA, Hussein MA, Jose J, Pinto R, Al-Omari A, Kharaba A et al: Corticosteroid Therapy for Critically III Patients with Middle East Respiratory Syndrome. Am J Respir Crit Care Med 2018, 197(6):757-767.

27. Stockman LJ, Bellamy R, Garner P: SARS: systematic review of treatment effects. PLoS Med 2006, 3(9):e343-e343.

28. Mahallawi WH, Khabour OF, Zhang Q, Makhdoum HM, Suliman BA: MERS-CoV infection in humans is associated with a pro-inflammatory Th1 and Th17 cytokine profile. Cytokine 2018, 104:8-13.

29. Wong CK, Lam CW, Wu AK, Ip WK, Lee NL, Chan IH, Lit LC, Hui DS, Chan MH, Chung SS et al: Plasma inflammatory cytokines and chemokines in severe acute respiratory syndrome. Clinical and experimental immunology 2004, 136(1):95-103.

\section{Tables}

Table 1: Demographics and clinical characteristics of 83 deaths with COVID-19 pneumonia 


\begin{tabular}{|ll|}
\hline Characteristics & Patients(n=83) \\
\hline Demographic factors & \\
\hline Age(years) Mean (SD), & $71.8(13.2)$ \\
\hline$<40$ & $2(2 \%)$ \\
\hline $40-49$ & $3(4 \%)$ \\
\hline $50-59$ & $10(12 \%)$ \\
\hline $60-69$ & $15(18 \%)$ \\
\hline $70-79$ & $27(33 \%)$ \\
\hline 380 & $26(31 \%)$ \\
\hline Sex & \\
\hline Men & $53(63.9 \%)$ \\
\hline Women & $30(36.1 \%)$ \\
\hline Clinical Characteristics & \\
\hline Signs and symptoms at onset & \\
\hline Fever & $78(94 \%)$ \\
\hline Myalgia or fatigue & $75(90.4 \%)$ \\
\hline Cough & $50(60.2 \%)$ \\
\hline pharyngalgia & $5(6 \%)$ \\
\hline Headache & $3(3.6 \%)$ \\
\hline Haemoptysis & $5(6 \%)$ \\
\hline Shortness of breath & $81(98.8 \%)$ \\
\hline Anorexia & $70(84.3 \%)$ \\
\hline Nausea or Vomiting & $2(2.4 \%)$ \\
\hline Diarrhoea & $4(4.8 \%)$ \\
\hline
\end{tabular}

Table 2: Key Epidemiologic Variables of 83 deaths with COVID-19 pneumonia 


\begin{tabular}{|ll|}
\hline Variable & Patients(n=83) \\
\hline Onset-to-admission interval & $10(7-14)$ \\
\hline $1-5 d$ & $11(13 \%)$ \\
\hline $6-10 d$ & \multicolumn{1}{|c|}{$36(43 \%)$} \\
\hline $10-15 d$ & $25(30 \%)$ \\
\hline 316 d & $11(13 \%)$ \\
\hline Onset-to-death interval & $17(14-21)$ \\
\hline Women & $16(14-20)$ \\
\hline $1-7 d$ & $1(3 \%)^{\dagger}$ \\
\hline $8-14 d$ & $8(27 \%)^{\dagger}$ \\
\hline $15-21 d$ & $15(50 \%)^{\dagger}$ \\
\hline $22-28 d$ & $4(13 \%)^{\dagger}$ \\
\hline $329 d$ & $2(7 \%)^{\dagger}$ \\
\hline Men & $18(14-23)$ \\
\hline $1-7 d$ & $2(4 \%)^{\square}$ \\
\hline $8-14 d$ & $13(25 \%)^{\square}$ \\
\hline $15-21 d$ & $24(45 \%)^{\square}$ \\
\hline $22-28 d$ & $11(21 \%)^{\square}$ \\
\hline $329 d$ & $3(6 \%)^{\square}$ \\
\hline
\end{tabular}

t, Proportion of women; ${ }^{\circledR}$, Proportion of men

Table 3: Characteristics of comorbidity of 83 deaths with COVID-19 pneumonia 


\begin{tabular}{|ll|}
\hline & death, $\mathrm{n}(\%)$ \\
\hline Comorbidities & $66(80)$ \\
\hline Hypertension & $47(57)$ \\
\hline Diabetes & $14(26)$ \\
\hline Cardiovascular & $26(31)$ \\
\hline Cerebrovascular disease & $14(17)$ \\
\hline Malignancy & $5(6)$ \\
\hline Chronic lung disease & $16(19.3)$ \\
\hline Chronic renal disease & $5(6)$ \\
\hline Chronic liver disease & $3(4)$ \\
\hline Number of comorbidities & \\
\hline 1.00 & $28(34)$ \\
\hline 2.00 & $17(21)$ \\
\hline 3.00 & $14(17)$ \\
\hline 4.00 & $6(7)$ \\
\hline 5.00 & $1(1)$ \\
\hline
\end{tabular}

Table 4: Laboratory findings of 83 deaths with COVID-19 pneumonia 


\begin{tabular}{|c|c|c|}
\hline & Normal Range & Patients $(n=83)$ \\
\hline White blood cell count, $\times 10 \otimes / L$ & $3.5-9.5$ & $9.1(4.7)$ \\
\hline Decreased & & $5(6 \%)$ \\
\hline Increased & & $34(41 \%)$ \\
\hline Neutrophil count, $\times 10 \otimes / L$ & $1.8-6.3$ & $6.9(4.4-11.5)$ \\
\hline Increased & & $49(59 \%)$ \\
\hline Lymphocyte count, $\times 10 \rrbracket / L$ & $1.1-3.2$ & $0.6(0.4-0.9)$ \\
\hline Decreased & & $69(83 \%)$ \\
\hline Mononuclear leucocyte, $\times 108 / L$ & $0.1-0.6$ & $0.4(0.3-0.5)$ \\
\hline Increased & & $16(19 \%)$ \\
\hline Haemoglobin ,g/L & Men $\bigotimes 130.0-175.0 \otimes W o m e n \bigotimes 115-150$ & $116(104-121)$ \\
\hline Decreased & & $34(41 \%)$ \\
\hline Platelets,$\times 10 \otimes / L$ & $125.0-350.0$ & $166(72)$ \\
\hline Decreased & & $24(29 \%)$ \\
\hline Total bilirubin,$\mu \mathrm{mol} / \mathrm{L}$ & $0.0-23.0$ & 14.1(9.8-19.9) \\
\hline Increased & & $16(19 \%)$ \\
\hline Direct bilirubin, $\mu \mathrm{mol} / \mathrm{L}$ & $0.0-8.0$ & $5.6(4.2-9.3)$ \\
\hline Increased & & $29(35 \%)$ \\
\hline Aspartate aminotransferase, $\mathrm{U} / \mathrm{L}$ & $15.0-40.0$ & $43(28-62)$ \\
\hline Increased & & $47(57 \%)$ \\
\hline Alanine aminotransferase, $\mathrm{U} / \mathrm{L}$ & $7.0-40.0$ & 25(19-49) \\
\hline Increased & & $25(30 \%)$ \\
\hline Alkaline phosphatase, U/L & $50-135$ & $76(59-105)$ \\
\hline Increased & & $12(14 \%)$ \\
\hline Gamma-glutamyl transpeptidase, U/L & $7-45$ & $44(23-75)$ \\
\hline Increased & & $41(49 \%)$ \\
\hline Albumin, $\mathrm{g} / \mathrm{L}$ & $40-55$ & $33.7(4.1)$ \\
\hline Decreased & & 77(93\%) \\
\hline Globulin, g/L & $20-40$ & $25.6(22.6-29.0)$ \\
\hline
\end{tabular}




\begin{tabular}{|ccc|}
\hline Decreased & & $4(5 \%)$ \\
\hline Increased & & $2(2 \%)$ \\
\hline Lactate dehydrogenase, $\mathrm{U} / \mathrm{L}$ & $120-250$ & $493(362-682)$ \\
\hline Increased & & $79(95 \%)$ \\
\hline serum creatinine,$\mu \mathrm{mol} / \mathrm{L}$ & $41-81$ & $77(55-113)$ \\
\hline Increased & & $39(47 \%)$ \\
\hline Blood urea nitrogen ,mmol/L & $3.1-8.8$ & $9.36(5.50-16.00)$ \\
\hline Increased & & $44(53 \%)$ \\
\hline Prothrombin time, $\mathrm{S}$ & $9.0-13.0$ & $12.9(12.2-14.2)$ \\
\hline Increased & & $36(43 \%)$ \\
\hline Activated partial thromboplastin time,s & $25.0-31.3$ & $29.1(27.1-32.5)$ \\
\hline Increased & & $27(33 \%)$ \\
\hline D-dimer, $\mathrm{mg} / \mathrm{L}$ & $0.0-0.55$ & $4.68(1.09-18.00)$ \\
\hline Increased & & $78(94 \%)$ \\
\hline Procalcitonin ,ng/mL & $0.0-0.1$ & $0.23(0.12-0.94)$ \\
\hline Increased & & $69(83 \%)$ \\
\hline C-reactive protein ,mg/mL & $0.0-10.0$ & $85(47-180.0)$ \\
\hline Increased & & $79(95 \%)$ \\
\hline
\end{tabular}

Table 5: Chest CT findings and treatment of 83 deaths with COVID-19 pneumonia 


\begin{tabular}{|c|c|}
\hline & Patients, n(\%) \\
\hline \multicolumn{2}{|l|}{ Chest CT characteristics } \\
\hline Unilateral pneumonia & $12(14.5)$ \\
\hline Bilateral pneumonia & $71(85.5)$ \\
\hline \multicolumn{2}{|l|}{ Treatment } \\
\hline Antifungal therapy & $2(2.4)$ \\
\hline Antibiotic therapy & $81(97.6)$ \\
\hline Glucocorticoid therapy & $54(65.1)$ \\
\hline Intravenous immunoglobulin therapy & $54(65.1)$ \\
\hline CRRT & $6(7.3)$ \\
\hline Invasive mechanical ventilation & $20(24.1)$ \\
\hline ECMO & $1(1.2)$ \\
\hline
\end{tabular}

Abbreviation: CRRT, continuous renal replacement therapy; ECMO, extracorporeal membrane oxygenation.

\section{Figures}




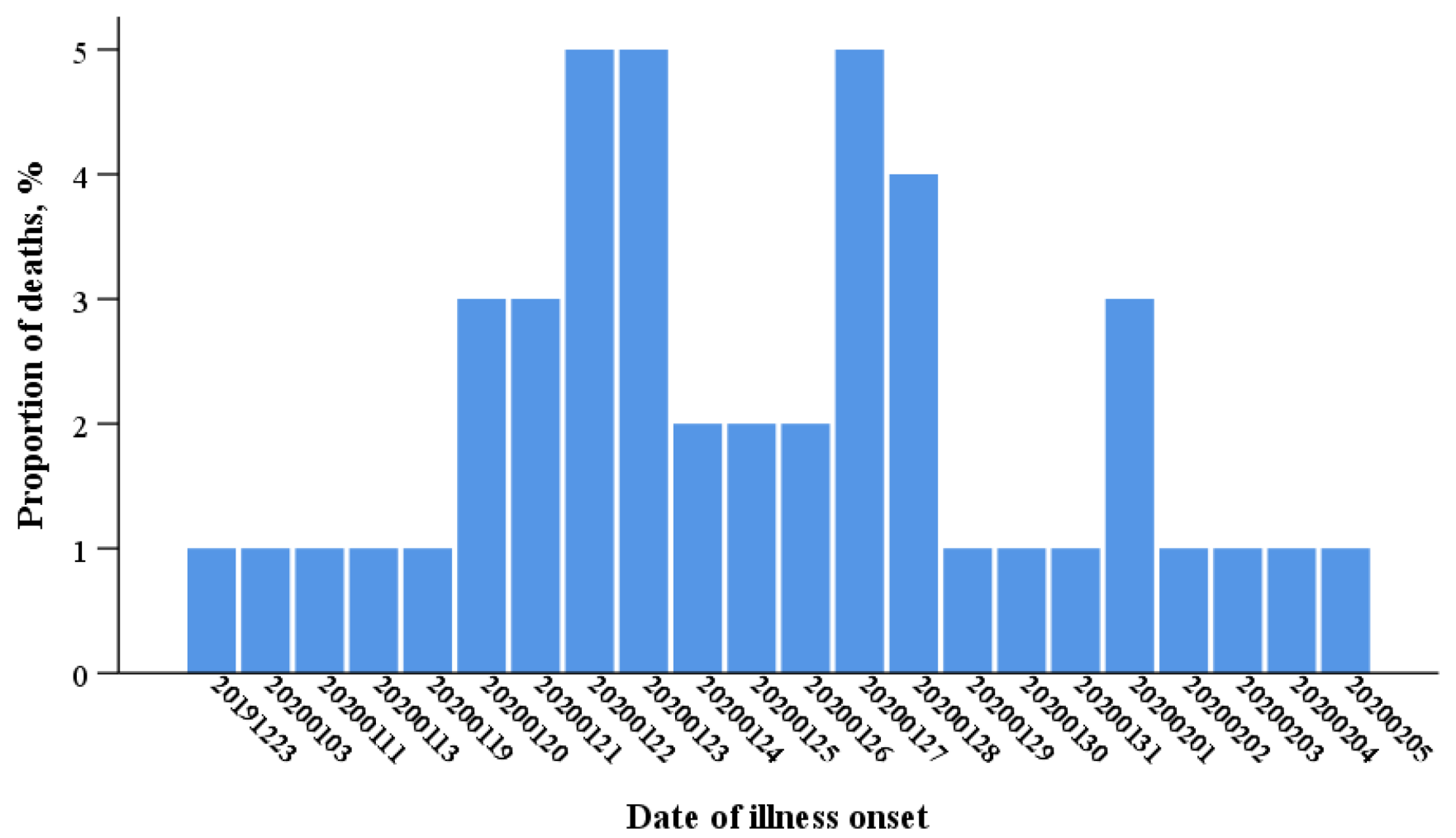

Figure 1. Date of illness onset of 83 deaths with COVID-19 pneumonia

Figure 1

Date of illness onset of 83 deaths with COVID-19 pneumonia 